\title{
Cranio-orbito-zygomatic approach for a previously coiled/ recurrent giant MCA aneurysm in a hybrid angio/OR suite
}

\author{
Leopold Arko, M.D., ${ }^{1}$ Eric Quach, B.S., ${ }^{2}$ Vishad Sukul, M.D., ${ }^{1}$ Anuj Desai, B.S., ${ }^{2}$ Kelly Gassie, B.S., ${ }^{3}$ \\ and Kadir Erkmen, M.D.1,2
}

'Department of Neurosurgery, Temple University Hospital; ${ }^{2}$ Temple University School of Medicine, Philadelphia, Pennsylvania; and ${ }^{3}$ Quillen College of Medicine, East Tennessee State University, Johnson City, Tennessee

\begin{abstract}
We present surgical clipping of a giant middle cerebral artery aneurysm. The patient is a 64-year-old woman who suffered subarachnoid hemorrhage in 2005. She was treated with coiling of the aneurysm at an outside institution. She presented to our clinic with headaches and was found on angiography to have giant recurrence of the aneurysm. To allow adequate exposure for clipping, we performed the surgery through a cranio-orbito-zygomatic (COZ) skull base approach, which is demonstrated. The surgery was performed in an operating room/angiography hybrid suite allowing for high quality intraoperative angiography. The technique and room flow are also demonstrated.
\end{abstract}

The video can be found here: http://youtu.be/eePcyOMi85M.

KEYWORDS COZ; cranio-orbito-zygomatic approach; skull base approach; hybrid angiography; video 